\title{
STATIONARY VALUES OF THE TRANSMISSION RATIO OF THE PLANAR FOURBAR
}

\author{
J. Wittenburg \\ Institute of Technical Mechanics, \\ University of Karlsruhe, Germany
}

\begin{abstract}
The transmission ratio of the planar fourbar, i.e. the ratio of the angular velocities of input link and output link, is a function of the input angle. Freudenstein [1] showed how to calculate stationary values of the transmission ratio. In the present paper a new method is described. Like Freudenstein's method it results in a sixth-order polynomial equation.
\end{abstract}

\section{INTRODUCTION}

The planar fourbar is composed of four links, namely the fixed link of length $\ell$, the input link of length $r_{1}$, the output link of length $r_{2}$ and the coupler of length $a$ (see the fourbar $\mathrm{A}_{0} \mathrm{ABB}_{0}$ in Fig. 1). The angles of rotation of input link and of output link relative to the fixed link, both positive counter-clockwise, are denoted $\varphi$ and $\psi$, respectively. The transfer function determines $\psi$ as function of $\varphi$. The time derivative of this function yields an expression for the transmission ratio $i=\dot{\varphi} / \dot{\psi}$ as function of $\varphi$. Subject of investigation are stationary values of $i(\varphi)$. In ref.[1] Freudenstein gave a sixth-order polynomial equation for a certain geometrical variable. The roots of this equation determine the input angles at which $i(\varphi)$ is stationary. In the present paper a new sixth-order polynomial equation with $\cos \varphi$ as variable is formulated. It is shown that the coefficients of this polynomial are invariant with respect to an interchange of $\ell$ and $r_{1}$. From this it follows that two fourbars with interchanged link lengths $\ell$ and $r_{1}$ have stationary transmission ratios $i_{\max }$ and $i_{\min }$, respectively, at identical input angles $\varphi$. Under certain conditions on the link lengths the polynomial is of fifth or of third or of second order. It is not shown in the present paper that also Freudenstein's equation can be written in a symmetrical form and that it leads, under the same conditions on link lengths, to fifth-, third- and second-order equations ${ }^{1}$.

\footnotetext{
${ }^{1}$ For relationships to Freudenstein's method see the monograph Wittenburg: Kinematics (to appear)
} 


\section{TRANSFER FUNCTION}

In the $x, y$-system shown in Fig. 1 the end points A and B of input link and output link have the coordinates

$$
\left.\begin{array}{ll}
x_{\mathrm{A}}=r_{1} \cos \varphi, & x_{\mathrm{B}}=\ell+r_{2} \cos \psi \\
y_{\mathrm{A}}=r_{1} \sin \varphi, & y_{\mathrm{B}}=r_{2} \sin \psi .
\end{array}\right\}
$$

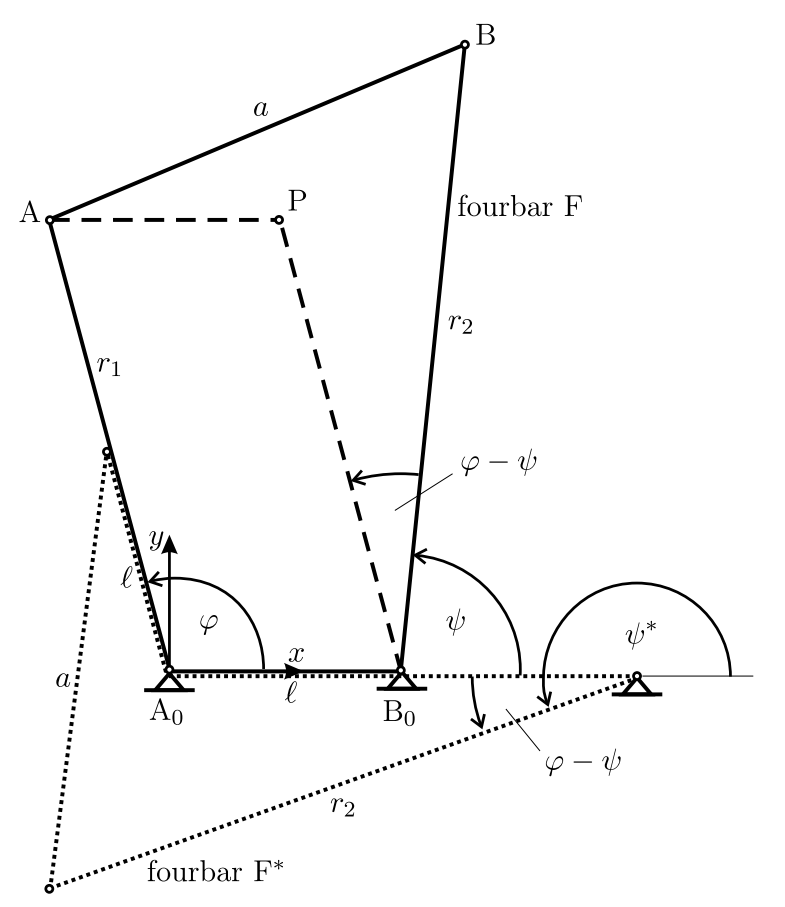

Fig. 1. Fourbar $\mathrm{F}$ and the associated fourbar $\mathrm{F}^{*}$ with link lengths $r_{1}$ and $\ell$ interchanged

The constant length $a$ of the coupler requires that $\left(x_{\mathrm{B}}-x_{\mathrm{A}}\right)^{2}+\left(y_{\mathrm{B}}-y_{\mathrm{A}}\right)^{2}=a^{2}$. This results in the transfer function $f(\varphi, \psi)=0$ :

$$
f=2 r_{2}\left(\ell-r_{1} \cos \varphi\right) \cos \psi-2 r_{1} r_{2} \sin \varphi \sin \psi-2 \ell r_{1} \cos \varphi+r_{1}^{2}+\ell^{2}+r_{2}^{2}-a^{2}=0
$$

or

$$
f=2 \ell r_{2} \cos \psi-2 \ell r_{1} \cos \varphi-2 r_{1} r_{2} \cos (\varphi-\psi)+r_{1}^{2}+\ell^{2}+r_{2}^{2}-a^{2}=0 .
$$

Equation (2) has the form

$$
A(\varphi) \cos \psi+B(\varphi) \sin \psi=C(\varphi)
$$

with coefficients

$$
A=2 r_{2}\left(\ell-r_{1} \cos \varphi\right), \quad B=-2 r_{1} r_{2} \sin \varphi, \quad C=2 r_{1} \ell \cos \varphi-\left(r_{1}^{2}+\ell^{2}+r_{2}^{2}-a^{2}\right) .
$$


For every angle $\varphi$ there exist two solutions $\psi_{1}$ and $\psi_{2}$. They are determined through their sines and cosines:

$$
\left.\begin{array}{l}
\cos \psi_{1,2}=\frac{A C \mp B \sqrt{A^{2}+B^{2}-C^{2}}}{A^{2}+B^{2}}, \\
\sin \psi_{1,2}=\frac{B C \pm A \sqrt{A^{2}+B^{2}-C^{2}}}{A^{2}+B^{2}} .
\end{array}\right\}
$$

These expressions depend on three parameters only, namely on $r_{1} / \ell, r_{2} / \ell$ and $a / \ell$. Equations (5) yield

$$
\begin{gathered}
A^{2}+B^{2}=4 r_{2}^{2}\left(\ell^{2}+r_{1}^{2}-2 r_{1} \ell \cos \varphi\right)=-4 r_{2}^{2}\left(C+r_{2}^{2}-a^{2}\right), \\
A^{2}+B^{2}-C^{2}=4 r_{2}^{2} a^{2}-\left(C+2 r_{2}^{2}\right)^{2}=-\left[C+2 r_{2}\left(a+r_{2}\right)\right]\left[C-2 r_{2}\left(a-r_{2}\right)\right] \\
=-\left[2 r_{1} \ell \cos \varphi-\left(r_{1}^{2}+\ell^{2}\right)+\left(r_{2}+a\right)^{2}\right]\left[2 r_{1} \ell \cos \varphi-\left(r_{1}^{2}+\ell^{2}\right)+\left(r_{2}-a\right)^{2}\right] .
\end{gathered}
$$

The angles $\psi_{1}$ and $\psi_{2}$ are real for all angles $\varphi$ satisfying the condition $A^{2}+B^{2}-C^{2} \geq 0$. Let $\phi$ denote all angles $\varphi$ for which the equality sign is valid. From Eq.(9) the cosines of these angles are obtained:

$$
\cos \phi_{1,2}=\frac{r_{1}^{2}+\ell^{2}-\left(r_{2} \mp a\right)^{2}}{2 r_{1} \ell} .
$$

These angles are limit angles of fourbars in which the input link cannot rotate full circle. The limit positions are characterized by collinearity of output link and coupler.

\section{INTERCHANGE OF INPUT LINK AND FIXED LINK}

In Fig. 1 the fourbar $\mathrm{A}_{0} \mathrm{ABB}_{0}$ with link lengths $\ell, r_{1}, a, r_{2}$ is called fourbar $\mathrm{F}$. Broken lines parallel to the fixed link and to the input link define the point $\mathrm{P}$. The quadrilateral $\mathrm{B}_{0} \mathrm{PAB}$ is drawn one more time in dotted lines. The dotted quadrilateral is called fourbar $\mathrm{F}^{*}$. Its fixed link has length $r_{1}$ and its input link has length $\ell$. Both fourbars have the same coupler and the same output link. If $\mathrm{F}$ is a foldable fourbar then also $\mathrm{F}^{*}$ is foldable. If $\mathrm{F}$ is a double-rocker of first kind (of second kind) then also $\mathrm{F}^{*}$ is a double-rocker of first kind (of second kind). If $\mathrm{F}$ is a double-crank then $\mathrm{F}^{*}$ is either a double-crank or a crank-rocker. If $\mathrm{F}$ is a crank-rocker then $\mathrm{F}^{*}$ is either a double-crank (if fixed link and crank are interchanged) or a crank-rocker (if fixed link and rocker are interchanged).

In Fig. $1 \mathrm{~F}$ and $\mathrm{F}^{*}$ have one and the same input angle $\varphi$. The relation between the output angles $\psi$ and $\psi^{*}$ is seen to be

$$
\psi+\psi^{*} \equiv \varphi+\pi .
$$

For a given angle $\varphi$ Eqs.(6) determine in the fourbar $\mathrm{F}$ two angles $\psi_{1}$ and $\psi_{2}$ and in the fourbar $\mathrm{F}^{*}$ with coefficients $A^{*}=2 r_{2}\left(r_{1}-\ell \cos \varphi\right), B^{*}=-2 \ell r_{2} \sin \varphi, C^{*}=C$ two angles $\psi_{1}^{*}$ and $\psi_{2}^{*}$. The coordination of these pairs of angles is as follows: $\psi_{1}+\psi_{2}^{*} \equiv \varphi+\pi$. This is verified by substituting $A, B, C$ and $A^{*}, B^{*}, C^{*}$ into the equation $\cos \psi_{1} \cos \psi_{2}^{*}-$ $\sin \psi_{1} \sin \psi_{2}^{*} \equiv-\cos \varphi$. 


\section{TRANSMISSION RATIO}

The angular velocity ratio $i=\dot{\varphi} / \dot{\psi}$ is called transmission ratio of the fourbar. An analytical expression for the ratio $1 / i$ is found by differentiating the transfer function $f(\varphi, \psi)=0$ with respect to time:

This yields

$$
\dot{\varphi} \frac{\partial f}{\partial \varphi}+\dot{\psi} \frac{\partial f}{\partial \psi}=0
$$

$$
\frac{1}{i}=\frac{\dot{\psi}}{\dot{\varphi}}=-\frac{\partial f}{\partial \varphi} / \frac{\partial f}{\partial \psi}
$$

Equation (3) yields

$$
\frac{\partial f}{\partial \varphi}=2 \ell r_{1} \sin \varphi+2 r_{1} r_{2} \sin (\varphi-\psi), \quad \frac{\partial f}{\partial \psi}=-2 \ell r_{2} \sin \psi-2 r_{1} r_{2} \sin (\varphi-\psi)
$$

whence follows

$$
\begin{aligned}
& \frac{1}{i}=\frac{r_{1}}{r_{2}} \\
& \times \frac{\ell \sin \varphi+r_{2} \sin (\varphi-\psi)}{\ell \sin \psi+r_{1} \sin (\varphi-\psi)}=\frac{r_{1}}{r_{2}} \frac{\ell \sin \varphi+r_{2}(\sin \varphi \cos \psi-\cos \varphi \sin \psi)}{\ell \sin \psi+r_{1}(\sin \varphi \cos \psi-\cos \varphi \sin \psi)}
\end{aligned}
$$

and with Eqs.(6)

$$
\begin{aligned}
& \frac{1}{i}=\frac{r_{1}}{r_{2}} \\
& \times \frac{\ell\left(A^{2}+B^{2}\right) \sin \varphi+r_{2}\left[(A \sin \varphi-B \cos \varphi) C \mp(B \sin \varphi+A \cos \varphi) \sqrt{A^{2}+B^{2}-C^{2}}\right]}{\ell\left(B C \pm A \sqrt{A^{2}+B^{2}-C^{2}}\right)+r_{1}\left[(A \sin \varphi-B \cos \varphi) C \mp(B \sin \varphi+A \cos \varphi) \sqrt{A^{2}+B^{2}-C^{2}}\right]} .
\end{aligned}
$$

From Eqs.(5) it follows that

$$
\left.\begin{array}{l}
A \sin \varphi-B \cos \varphi=2 r_{2} \ell \sin \varphi \\
B \sin \varphi+A \cos \varphi=2 r_{2}\left(\ell \cos \varphi-r_{1}\right) \\
\ell B+r_{1}(A \sin \varphi-B \cos \varphi)=0
\end{array}\right\}
$$

These equations in combination with Eqs.(7) and (8) yield the final formula

$$
\frac{2}{i}=\frac{\left(\cos \varphi-p_{1}\right) \sqrt{\lambda^{2}-\left(\cos \varphi-p_{4}\right)^{2}} \pm\left(\cos \varphi-p_{3}\right) \sin \varphi}{\left(\cos \varphi-p_{2}\right) \sqrt{\lambda^{2}-\left(\cos \varphi-p_{4}\right)^{2}}}
$$

with dimensionless constants

$$
\lambda=\frac{r_{2} a}{r_{1} \ell}, \quad p_{1}=\frac{r_{1}}{\ell}, \quad p_{2}=\frac{r_{1}^{2}+\ell^{2}}{2 r_{1} \ell}, \quad p_{3}=p_{2}-\frac{r_{2}^{2}-a^{2}}{2 r_{1} \ell}, \quad p_{4}=p_{2}-\frac{r_{2}^{2}+a^{2}}{2 r_{1} \ell} .
$$

These constants are related as follows:

$$
p_{2}^{2}-1=\left(p_{1}-p_{2}\right)^{2}, \quad\left(p_{4}-p_{2}\right)^{2}-\lambda^{2}=\left(p_{3}-p_{2}\right)^{2} .
$$

From the first relation in combination with the definition of $p_{2}$ it follows that $p_{2} \geq 1$. The equality sign applies if and only if $r_{1}=\ell$ and with this $p_{1}=p_{2}=1$. This, in turn, has the consequence that the denominator expression $\cos \varphi-p_{2}$ in Eq.(18) is zero only 
if the two conditions $\varphi=0$ and $r_{1}=\ell$ are satisfied which implies that also $r_{2}=a$. This indicates the folding position of a rhomboid fourbar. The square root in the denominator of Eq.(18) is zero only if $\varphi$ is one of the limit angles $\phi_{1,2}$ of the input link which must then be a rocker (see Eq.(10)).

With the exception of $p_{1}$ all constants in Eqs.(19) are invariant with respect to an interchange of base length $\ell$ and input link length $r_{1}$. Because of the first Eq.(20) this is true also for $\left(p_{1}-p_{2}\right)^{2}$. A relation between the ratios $1 / i$ and $1 / i^{*}$ of the two fourbars with interchanged link lengths is obtained by differentiating the identity Eq.(11) with respect to time:

$$
\frac{1}{i}+\frac{1}{i^{*}}=\frac{\dot{\psi}}{\dot{\varphi}}+\frac{\dot{\psi}^{*}}{\dot{\varphi}} \equiv 1
$$

\section{STATIONARY VALUES OF TRANSMISSION RATIO}

Let $P_{1}$ be the center of rotation of the output link relative to the input link, and let $P_{2}$ be the center of rotation of the coupler relative to the fixed link. The former is the point of intersection of coupler and fixed link (also referred to as base line), and the latter is the point of intersection of output link and input link. Freudenstein [1] discovered that a stationary value of the transmission ratio occurs in positions in which the line $\overline{\mathrm{P}}_{1} \mathrm{P}_{2}$ is orthogonal to the coupler. Based on this general rule Freudenstein developed an analytical method for the determination of the associated angles $\varphi$. In the present paper Freudenstein's rule is applied only to the special case that a stationary value occurs at $\varphi=0$ or at $\varphi=\pi$. In these cases, $\mathrm{P}_{1}$ and $\mathrm{P}_{2}$ are located on the base line, and the coupler is orthogonal to the base line. Then, the parameters satisfy the condition

$$
\left.\begin{array}{ll}
\text { stationary value at } \varphi=0: & \left(\ell-r_{1}\right)^{2}+a^{2}=r_{2}^{2} \\
\text { stationary value at } \varphi=\pi: & \left(\ell+r_{1}\right)^{2}+a^{2}=r_{2}^{2} .
\end{array}\right\}
$$

The new method is based on Eq.(18). With the abbreviation $x=\cos \varphi$ this equation is written in the form

$$
\frac{2}{i(x)}=\frac{\left(x-p_{1}\right) P \pm\left(x-p_{3}\right) Q}{\left(x-p_{2}\right) P}, \quad P=\sqrt{\lambda^{2}-\left(x-p_{4}\right)^{2}}, \quad Q=\sqrt{1-x^{2}} .
$$

The stationarity condition $\mathrm{d}(1 / i) / \mathrm{d} x=0$ has the form (the prime denotes the derivative with respect to $x$ )

$$
\mp\left(p_{1}-p_{2}\right) P^{2}=\left(p_{3}-p_{2}\right) P Q+\left(x-p_{2}\right)\left(x-p_{3}\right)\left(P Q^{\prime}-Q P^{\prime}\right) .
$$

Now, $P^{\prime}=-\left(x-p_{4}\right) / P$ and $Q^{\prime}=-x / Q$ are substituted. The resulting equation is multiplied by $P Q$. This eliminates the case $\sin \varphi=0$. Whether this is a solution is checked with Eq.(22). After this multiplication the equation has the form

$$
\begin{aligned}
& \pm\left(p_{1}-p_{2}\right)\left[\left(x-p_{4}\right)^{2}-\lambda^{2}\right] \sqrt{\left(x^{2}-1\right)\left[\left(x-p_{4}\right)^{2}-\lambda^{2}\right]} \\
& =\left(p_{3}-p_{2}\right)\left(x^{2}-1\right)\left[\left(x-p_{4}\right)^{2}-\lambda^{2}\right] \\
& -\left(x-p_{2}\right)\left(x-p_{3}\right)\left[p_{4}\left(1+x^{2}\right)+x\left(\lambda^{2}-p_{4}^{2}-1\right)\right] .
\end{aligned}
$$


The special case $r_{1}=\ell$ is characterized by $p_{1}=p_{2}=1$ and, therefore, by the third-order equation

$$
\left(p_{3}-1\right)(1+x)\left[\lambda^{2}-\left(x-p_{4}\right)^{2}\right]+\left(x-p_{3}\right)\left[p_{4}\left(1+x^{2}\right)+x\left(\lambda^{2}-p_{4}^{2}-1\right)\right]=0 .
$$

The equation is quadratic if, in addition, also $a=\ell$.

In the general case $r_{1} \neq \ell$ Eq.(25) is squared. The squared equation is invariant with respect to the interchange of $r_{1}$ and $\ell$ (see the comments following Eqs.(19) and (20)). Because of the sign \pm no extraneous roots are introduced by squaring. Equation (25) with the positive sign has the meaningless root $x=p_{2}>1$. This is verified with the help of Eqs.(20). From this it follows that the squared equation is divisible by $\left(x-p_{2}\right)^{2}$. Following this division it is a sixth-order equation. The division is performed in two steps. Squaring results in the equation

$$
\begin{aligned}
& \left(x^{2}-1\right)\left[\left(x-p_{4}\right)^{2}-\lambda^{2}\right]^{2}\left\{\left(p_{1}-p_{2}\right)^{2}\left[\left(x-p_{4}\right)^{2}-\lambda^{2}\right]-\left(p_{3}-p_{2}\right)^{2}\left(x^{2}-1\right)\right\} \\
= & \left(x-p_{2}\right) F(x)\left\{\left(x-p_{2}\right) F(x)-2\left(p_{3}-p_{2}\right)\left(x^{2}-1\right)\left[\left(x-p_{4}\right)^{2}-\lambda^{2}\right]\right\}
\end{aligned}
$$

with the third-order polynomial

$$
F(x)=\left(x-p_{3}\right)\left[p_{4}\left(1+x^{2}\right)+x\left(\lambda^{2}-p_{4}^{2}-1\right)\right] .
$$

Taking into account Eqs.(20) the expression in curled brackets on the left-hand side is written in the form $\left(x-p_{2}\right)(A x+B)$ with constants

$$
A=\left(p_{1}-p_{2}\right)^{2}-\left(p_{3}-p_{2}\right)^{2}, \quad B=p_{2} A-2 p_{4}\left(p_{1}-p_{2}\right)^{2} .
$$

Thus, division of Eq.(27) by $\left(x-p_{2}\right)$ produces the equation

$$
\begin{aligned}
& \left(x^{2}-1\right)\left[\left(x-p_{4}\right)^{2}-\lambda^{2}\right]\left\{\left[\left(x-p_{4}\right)^{2}-\lambda^{2}\right](A x+B)+2\left(p_{3}-p_{2}\right) F(x)\right\} \\
& =\left(x-p_{2}\right) F^{2}(x) .
\end{aligned}
$$

The expression in curled brackets is a third-order polynomial $K_{3} x^{3}+K_{2} x^{2}+K_{1} x+K_{0}$ with coefficients

$$
\left.\begin{array}{l}
K_{3}=A+2 p_{4}\left(p_{3}-p_{2}\right), \\
K_{2}=B-2 p_{4} A+2\left(p_{3}-p_{2}\right)\left(\lambda^{2}-p_{4}^{2}-1-p_{3} p_{4}\right), \\
K_{1}=-2 p_{4} B+A\left(p_{4}^{2}-\lambda^{2}\right)+2\left(p_{3}-p_{2}\right)\left[p_{4}-p_{3}\left(\lambda^{2}-p_{4}^{2}-1\right)\right] .
\end{array}\right\}
$$

Division by $\left(x-p_{2}\right)$ produces the second-order polynomial $K_{3} x^{2}+\left(x+p_{2}\right)\left(K_{2}+p_{2} K_{3}\right)+$ $K_{1}$. With this expression Eq.(30) yields the desired sixth-order equation

$$
\left(x^{2}-1\right)\left[\left(x-p_{4}\right)^{2}-\lambda^{2}\right]\left[K_{3} x^{2}+\left(x+p_{2}\right)\left(K_{2}+p_{2} K_{3}\right)+K_{1}\right]-F^{2}(x)=0 .
$$

The coefficient of $x^{6}$ is

$$
K_{3}-p_{4}^{2}=\left(p_{1}-p_{2}\right)^{2}-\left(p_{3}-p_{2}-p_{4}\right)^{2}=\frac{\left(\ell^{2}-a^{2}\right)\left(a^{2}-r_{1}^{2}\right)}{\left(r_{1} \ell\right)^{2}} .
$$

From this it follows that the equation is of fifth order if $a=\ell$ and/or $a=r_{1}$. Only real roots $|x| \leq 1$ are significant. For every such root it is checked to which sign in Eq.(25) the root belongs. With the same sign Eqs.(23) and (6) determine the corresponding stationary value of $1 / i$ and the angle $\psi$. Like Freudenstein's method also this method does not make any statement about the number of stationary values. 
Example: A fourbar with parameters $\ell=3, r_{1}=5, a=7$ and $r_{2}=6$ is a double-crank, and a fourbar with parameters $\ell=5, r_{1}=3, a=7$ and $r_{2}=6$ ( $\ell$ and $r_{1}$ interchanged) is a crank-rocker. With both sets of parameters Eq.(32) has the four real roots $x=\cos \varphi \approx-0.084,0.9882,1.11$ and 4.02 . The first two roots determine the angles $\varphi \approx 94.8^{\circ}$ and $\varphi \approx 8.8^{\circ}$, respectively (arbitrarily positive, because to every position $(\varphi, \psi)$ exists the symmetric position $(-\varphi,-\psi))$. For the double-crank Eq.(25) is satisfied with the lower sign for $x \approx-0.084$ and with the upper sign for $x \approx 0.9882$. From this it follows that the double-crank has the two positions of stationarity $\left(\varphi \approx 94.8^{\circ}, \psi_{2} \approx 195.5^{\circ}\right)$ with $(1 / i)_{\min } \approx 0.42$ and $\left(\varphi \approx 8.8^{\circ}, \psi_{1} \approx-88.6^{\circ}\right)$ with $(1 / i)_{\max } \approx 2.7$. For the crank-rocker the two positions of stationarity are $\left(\varphi \approx 94.8^{\circ}, \psi_{1} \approx\right.$ $\left.79.3^{\circ}\right)$ with $(1 / i)_{\max } \approx 1-0.42=0.58$ and $\left(\varphi \approx 8.8^{\circ}, \psi_{2} \approx-82.5^{\circ}\right)$ with $(1 / i)_{\min } \approx$ $1-2.7=-1.7$. It may be checked that in all four positions the line $\overline{\mathrm{P}}_{1} \mathrm{P}_{2}$ is orthogonal to the coupler. End of example.

Summary: The inverse of the transmission ratio $i=\dot{\varphi} / \dot{\psi}$ of the planar fourbar is formulated as function of $x=\cos \varphi$. The stationarity condition $\mathrm{d}(1 / i) / \mathrm{d} x=0$ is a sixth-order polynomial equation. Its roots are invariant with respect to an interchange of the link lengths $\ell$ (fixed link) and $r_{1}$ (input link). This has the consequence that two fourbars with interchanged link lengths $\ell$ and $r_{1}$ have stationary transmission ratios $i_{\text {stat }}$ and $i_{\text {stat }}^{*}$ at identical input angles $\varphi$. Moreover, the relationship between the two is $1 / i_{\text {stat }}+1 / i_{\text {stat }}^{*}=1$.

\section{REFERENCES}

[1] Freudenstein F (1956) On the maximum and minimum velocities and the accelerations in four-link mechanisms. Trans. ASME 78:779-787.

Received April 21, 2009

\section{CÁC GIÁ TRI DỬNG CỦA TỶ SỐ TRUYÈ̀N ĐỘNG CỦA HỆ BỐN THANH PHẲNG}

Tỷ số truyền động của hệ bốn thanh phẳng, tức là tỷ số của các vận tốc góc giữa các liên kết đầu vào và đầu ra, là một hàm của góc đầu vào. Ông Freudenstein [1] đã chỉ ra cách tính các giá trị dừng của tỷ số truyền động. Trong bài báo này một phương pháp mới sẽ được trình bầy. Cũng giống phương pháp của Freudenstein, nó đưa đến một phương trình đa thức bậc 6 . 